\title{
Cirugía cardiaca en ancianos Epidemiología, calidad de vida y funcionalidad postoperatoria
}

\author{
Heart surgery in the elderly \\ Epidemiology, quality of life and postoperative functionality \\ Edwin H. Etayo, Fernando I. González, María C. Florián, \\ Arnoby Chacón • Manizales (Colombia)
}

\section{Resumen}

Objetivo: describir la epidemiología de los pacientes llevados a cirugía cardiaca en el periodo entre 2004 y 2008, y valorar la funcionalidad y la calidad de vida postoperatoria en un subgrupo.

Material y métodos: estudio descriptivo de corte transversal. Análisis estadístico se realizó en epiinfo 2000 versión 3.5.3 de 2011 y una p < 0.05 se consideró estadísticamente significativa. La calidad de vida se evaluó con SF-12v2 y se aplicaron la escala de Barthel y de Lawton para la valoración de la funcionalidad.

Resultados: el promedio de edad fue 68.6 años, $2.4 \% \geq 80$ años. La mortalidad global hospitalaria fue $10.3 \%$ (9.2\% para revascularización, $11.1 \%$ para cambio valvular y del $16.7 \%$ para cirugía mixta). El promedio del componente de salud física fue 43.7 vs 49.6 (población de referencia), y el del componente de salud mental fue 53.2 vs 49.3. 79.4\% mejoran la autopercepción en salud posterior a la cirugía, $94.1 \%$ eran independientes en su $\mathrm{ABC}$ físico y $85.3 \%$ requerían alguna ayuda en sus actividades instrumentales.

Conclusiones: es el primer estudio en nuestro medio que describe la epidemiología de un grupo de pacientes ancianos llevados a cirugía cardiaca y que evalúa calidad de vida y funcionalidad; se evidenció una repercusión positiva en la calidad de vida, funcionalidad y en la autopercepción de salud. A nivel instrumental se evidenció mayor compromiso de las actividades que requieren movilidad y participación social, siendo el sexo femenino y la edad avanzada los factores de mayor impacto negativo. (Acta Med Colomb 2014; 39: 118-123).

Palabras clave: cirugía cardiaca, ancianos, calidad de vida, funcionalidad, autopercepción de salud.

\footnotetext{
Abstract

Objective: to describe the epidemiology of patients undergoing cardiac surgery in the period 2004 to 2008 and evaluate the functionality and postoperative quality of life in a subgroup.

Material and methods: a descriptive cross-sectional study . Statistical analysis was performed in Epi Info 2000 version 3.5.3 2011 and p < 0.05 was considered statistically significant. Quality of life was assessed by SF-12v2 and Barthel and Lawton scale for assessing the functionality was applied.

Results: mean age was 68.6 years, $2.4 \% \geq 80$ years. The overall hospital mortality was $10.3 \%(9.2$ $\%$ for revascularization, valve replacement $11.1 \%$ and $16.7 \%$ for joint surgery). The average physical health component was 43.7 vs 49.6 ( reference population) and the mental health component was 53.2 vs 49.3.79.4\% improve health self-perception after surgery, $94.1 \%$ were independent in their physical $\mathrm{ABC}$ and $85.3 \%$ required some help in their instrumental activities.

Conclusions: this is the first study in our environment describing the epidemiology of a group of elderly patients undergoing cardiac surgery that evaluates quality of life and functionality. A positive impact on quality of life, functionality and health self-perception was evidenced . At instrumental level, greater commitment of activities requiring mobility and social participation was evident. Female sex and advanced age were factors with greater negative impact. (Acta Med Colomb 2014; 39: 118-123).
}

Keywords: cardiac surgery, elderly, quality of life, function, health self-perception .
Dres. Edwin H. Etayo y Fernando I. González: Medicina interna y Geriatría, Universidad de Caldas; Dra. María C. Florián: Internista Intensivista, Profesora Postgrado de Medicina Interna, Jefe UCI Hospital Santa Sofía de Caldas; Dr. Arnoby Chacón: Epidemiólogo, Profesor Postgrado de Medicina Interna, Universidad de Caldas. Manizales (Colombia) Correspondencia: Edwin Harvey Etayo Ruiz. Manizales (Colombia) E-mail: edwin.etayo@gmail.com Recibido: 16/IV2013 Aceptado: 03/III/2014 


\section{Introducción}

Dentro del proceso de transición demográfica, el envejecimiento de la población colombiana plantea importantes desafíos sociales, económicos y en salud. En el último censo se evidenció un crecimiento poblacional de $39.27 \%$ durante las últimas dos décadas (1), siendo los mayores de 60 años el segmento de mayor crecimiento, grupo en quienes las enfermedades crónicas cardiovasculares tienen mayor incidencia requiriendo para su control intervenciones no sólo médicas sino también quirúrgicas, dentro de ellas la cirugía del aparato cardiovascular (2-4).

Los avances en las técnicas quirúrgicas y en los cuidados perioperatorios han permitido mejorar los resultados de la cirugía cardiaca en términos de morbilidad y mortalidad (4, $5)$. En consecuencia, la edad de los pacientes intervenidos se ha incrementado significativamente en los últimos años (4).

En geriatría se plantea un abordaje integral y los resultados de cualquier tratamiento se valoran también en términos de calidad de vida, funcionalidad y participación social (5, 6). El impacto en supervivencia ya no es el objetivo primario. La principal indicación para someter un paciente octogenario a una cirugía de corazón abierto seria: síntomas relacionados con la enfermedad de base que estén deteriorando su calidad de vida y que sean corregibles con la cirugía (4-6).

En nuestro medio no contamos con estudios que describan las características de los pacientes operados a corazón abierto, y mucho menos que valoren la calidad de vida y su funcionalidad postoperatoria; por esto el objetivo principal de este estudio es describir la epidemiología de una cohorte de pacientes mayores de 60 años, sometidos a cirugía cardiovascular y valorar la funcionalidad y calidad de vida de un subgrupo.

\section{Población de estudio}

\section{Material y métodos}

Todos los pacientes de $\geq 60$ años sometidos a cirugía cardiovascular en el Hospital Departamental Santa Sofía de Caldas E.S.E., Manizales, Colombia en el periodo comprendido entre enero de 2004 y diciembre de 2008.

\section{Recolección de datos}

Las fuentes de recolección fueron la historia clínica en físico y la historia clínica sistematizada, la valoración preanestésica y la base de datos del grupo quirúrgico.

Se realizó un formato de recolección de datos, el cual previamente se sometió a una prueba piloto; las variables preoperatorias recogidas fueron: edad, sexo, estado civil, nivel de escolaridad, sitio de residencia. Como comorbilidades se describieron: hipertensión arterial, dislipidemia, diabetes mellitus, tabaquismo, enfermedad pulmonar obstructiva crónica, falla cardiaca, enfermedad vascular periférica, insuficiencia renal previa (creatinina $>2 \mathrm{mg} / \mathrm{dL}$ ), accidente cerebrovascular (ACV), enfermedad carotídea, hipotiroidismo, infarto de miocardio (IAM) previo, la presencia de angina, el uso balón intraaórtico de contrapulsación (BIA) previo a la cirugía, el antecedente de intervención cardiaca o de procedimiento endovascular previo (angioplastia más stent). Se registró la fracción de eyección prequirúrgica, el ritmo cardiaco al ingreso, la anatomía coronaria, la presencia de lesión valvular y la función ventricular izquierda, así como la clase funcional de acuerdo con la New York Heart Association (NYHA) y el EUROSCORE de acuerdo con los datos del registro preanestésico.

Los factores operatorios analizados incluyeron: prioridad de la cirugía, tipo de intervención, número de coronarias revascularizadas, la válvula intervenida y el tipo de reemplazo utilizado; tiempos de intervención, circulación extracorpórea (CEC) y pinzamiento aórtico.

Se describieron la presencia de las siguientes complicaciones postoperatorias: sangrado precoz que precisó reintervención, taponamiento cardiaco, infarto del miocardio perioperatorio (aparición de una nueva onda Q patológica en el electrocardiograma o elevación del segmento ST y marcadores de daño miocárdico), bajo gasto cardiaco (necesidad de inotrópicos durante más de 48 horas), sepsis, neumonía, mediastinitis, infección de safenectomía, infección de catéter central, infección de vías urinarias, isquemia intestinal, insuficiencia respiratoria (ventilación mecánica más de 48 horas), ACV (déficit neurológico temporal o permanente), insuficiencia renal con o sin requerimiento dialítico, fibrilación auricular (FA), marcapasos definitivo. Se definió la mortalidad precoz como la ocurrida durante la hospitalización o en los primeros 30 días posterior al alta. Además se evaluaron los tiempos de estancia en UCI y en hospitalización.

Se evaluó la calidad de vida y la funcionalidad en el subgrupo de 97 pacientes operados en 2008, del cual 85 sobrevivieron logrando la evaluación de 34 de ellos; 17 mujeres y 17 hombres. Para el análisis de calidad de vida se aplicó Short Form 12 Health Survey version 2 (SF-12v2) válido al español (7) y aplicado en Colombia (8), disponible como programa de demostración en internet, evaluando los promedios del componente de salud física y salud mental y comparándolos con los promedios de una población de referencia (9). Adicionalmente la funcionalidad se valoró con escala de Barthel para el ABC físico y con escala de Lawton modificado versión (8-30 puntos) para $A B C$ instrumental ampliamente utilizadas en nuestro medio $(10,11)$.

\section{Análisis estadístico}

El análisis de los datos se realizó en el programa epiinfo 2000 versión 3.5.3 de 2011, un valor de p menor de 0,05 con IC de $95 \%$ se consideró estadísticamente significativo. Las variables continuas fueron descritas con promedio (rango), moda y mediana; se aplicó la prueba de chi $^{2}$ y el test exacto de Fisher según correspondiera para las variables dicotómicas y el test de ANOVA para comprobar la homogeneidad de la muestra. Se determinó la frecuencia de las diferentes comorbilidades, complicaciones postoperatorias y se calculó 
el odds ratio (OR) para identificar las variables que se relacionaron con la mortalidad (IC 95\%).

\section{Resultados}

De 419 historias clínicas se revisaron 417, dos historias no fueron encontradas. Los datos registrados en la historia clínica nos permitieron encontrar los siguientes resultados: $62.5 \%$ de los pacientes eran de sexo masculino con una relación hombre: mujer de 1.6; 58.2\% estaban en el rango de edad de 60-69 años, 39.4\% entre 70 y 79 años y sólo el $2.4 \%$ eran $\geq 80$ años, con un promedio de edad de 68.6 (rango 60-83) y una moda de 62 años.

Las comorbilidades y otras variables preoperatorias se presentan en la Tabla 1.

La diabetes predominó en las mujeres ( $\mathrm{p}<0.001)$ y en hombres predominaban la enfermedad renal crónica previa $(\mathrm{p}<0.0008)$ y tabaquismo $(\mathrm{p}<0.001)$.

El $64.5 \%$ de los pacientes tenían algún grado de compromiso de la función del ventrículo izquierdo (FEVI < 50\%) y el $16.9 \%$ tenía un compromiso severo (FEVI < 30\%) con un promedio porcentual de 49 y una moda de $60 \%$. Con respecto a la clase funcional medida por NYHA, $85 \%$ de los pacientes tenían algún grado de compromiso, $44.9 \%$ NYHA II, $28.9 \%$ NYHA III y $11.2 \%$ estaban en NYHA IV.

El $13.1 \%$ de las cirugías se realizaron con criterio de urgencia (clasificado así en el récord de anestesia).

Doscientos setenta y nueve pacientes fueron sometidos a cirugía de revascularización miocárdica, 108 a cambio valvular y 30 sometidos a cirugía mixta (revascularización y cambio valvular).

Se realizaron 108 procedimientos valvulares en 107 pacientes, sólo un paciente fue sometido a doble cambio valvular; la cirugía valvular aórtica fue la más común $54.2 \%$ vs $48.9 \%$ mitral y en $67.3 \%$ se utilizaron válvulas biológicas. Treinta y cinco procedimientos fueron realizados fuera de bomba (off pump) equivalente a $12.5 \%$ de todos los pacientes revascularizados. El tiempo promedio de circulación extracorpórea (CEC) fue de 100.8 minutos (rango 15-364 minutos) y la moda fue 90 minutos; el $22.9 \%$ fueron tiempos de CEC prolongados; el tiempo promedio de pinza aórtica fue 72 minutos (rango 7-269 minutos) y una moda de 47 minutos. Las complicaciones postoperatorias se resumen en las Figuras 1 y 2.

Las complicaciones POP que se relacionaron con mortalidad fueron el sangrado precoz que requirió intervención, el infarto del miocardio perioperatorio, el bajo gasto cardiaco, la sepsis, la insuficiencia respiratoria y la insuficiencia renal que requirió diálisis.

La tasa de letalidad fue de $10.3 \%$ pacientes con una distribución de la mortalidad por años así como se muestra en la Figura 3.

Las principales variables relacionadas con mortalidad fueron la edad $\geq 80$ años ( $\mathrm{p}=0.04)$, la cirugía de urgencia $(\mathrm{p}<0.001)$ y las complicaciones POP que se muestran en la Tabla 2 con su respectivo OR (IC 95\%).
Tabla 1. Variables preoperatorias.

\begin{tabular}{|c|c|}
\hline & $\mathrm{N}=417(\%)$ \\
\hline Edad en años (promedio) & 68,6 \\
\hline $60-69$ & 58.2 \\
\hline $70-79$ & 39.4 \\
\hline$\geq 80$ & 2.4 \\
\hline Masculino & 62.5 \\
\hline HTA & 77.3 \\
\hline Diabetes mellitus & 20.8 \\
\hline Dislipidemia & 60.9 \\
\hline Tabaquismo & 56.6 \\
\hline Falla cardiaca & 22.4 \\
\hline EPOC & 17.4 \\
\hline ERC previa & 6.6 \\
\hline Enfermedad carotídea & 2.6 \\
\hline EVP & 3.1 \\
\hline ECV previo & 4.1 \\
\hline Hipotiroidismo & 20.5 \\
\hline IAM previo & 46.8 \\
\hline Angina previa a cirugía & 49.4 \\
\hline Cx cardiaca previa & 5.5 \\
\hline $\mathrm{ACTP}+$ stent & 9.1 \\
\hline BCIA & 5.6 \\
\hline RS ingreso & 77.1 \\
\hline \multicolumn{2}{|c|}{$\begin{array}{l}\text { HTA: hipertensión arterial. EPOC: enfermedad pulmonar obstructiva crónica, ERC: } \\
\text { enfermedad renal crónica, EVP: enfermedad vascular periférica, ECV: evento cere- } \\
\text { brovascular, IM: infarto de miocardio, ACTP: angioplastia coronaria transluminal } \\
\text { percutánea, BCIA: balón de contrapulsación intraaórtico, RS: ritmo sinusal. }\end{array}$} \\
\hline
\end{tabular}

La mortalidad en relación con el tipo de cirugía se comportó así: para los pacientes sometidos a cirugía de revascularización miocárdica $9.2 \%$; para los valvulares $11.1 \%$ y para los de cirugía mixta (válvula y revascularización) $16.7 \%$. Sin embargo estos porcentajes estuvieron por debajo de la mortalidad calculada por escala de EUROSCORE tal como se muestra en la Figura 4.

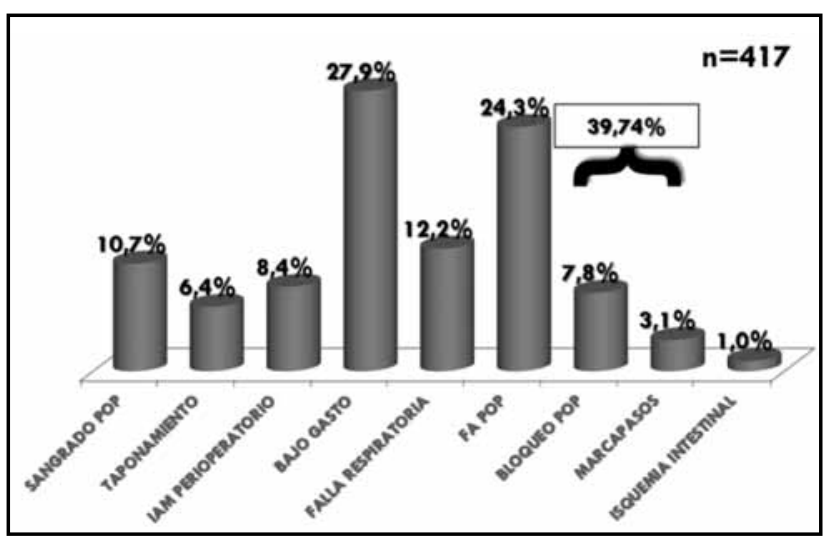

Figura 1. Complicaciones postoperatorias; FA=fibrilación auricular, $P O P=$ postoperatorio 
Tabla 2. Variables relacionadas con mortalidad.

\begin{tabular}{|l|c|c|c|c|}
\hline & No. & P & OR & IC \\
\hline Sangrado & 12 & 0.0000 & 4.1 & $1.9-8.8$ \\
Taponamiento & 4 & 0.3 & 1.6 & $0.5-5.0$ \\
IAM perioperatorio & 11 & 0.000 & 5.0 & $2.2-11.1$ \\
Bajo gasto & 27 & 0.0000 & 5.3 & $2.7-10.3$ \\
F. respiratoria & 12 & 0.001 & 3.4 & $1.6-7.2$ \\
FA POP & 11 & 0.8 & 1.06 & $0.5-2.2$ \\
Bloqueo & 8 & 0.006 & 3.1 & $1.3-7.6$ \\
Marcapaso & 0 & 0.2 & 0.0 & - \\
Isquemia intestinal & 2 & 0.05 & 9.0 & $1.2-66.0$ \\
F. renal con diálisis & 8 & 0.0000 & 11.9 & $4.1-35.0$ \\
F. renal sin diálisis & 13 & 0.003 & 2.8 & $1.3-5.7$ \\
ECV Post & 1 & 0.5 & 1.4 & $0.17-12.4$ \\
Inf. Safenectomía & 1 & 0.4 & 0.47 & $0.06-3.6$ \\
Inf. Cateter central & 2 & 0.11 & 4.5 & $0.8-25.3$ \\
IVU & 6 & 0.08 & 2.2 & $0.8-5.8$ \\
Mediastinitis & 6 & 0.08 & 2.2 & $0.8-58$ \\
Sepsis & 12 & 0.0000 & 4.9 & $2.2-10.7$ \\
Neumonía & 0.5 & 1.09 & $0.3-3.7$ \\
\hline OR. & 13 & 12 & & \\
\hline
\end{tabular}

OR: odds ratio, $\mathrm{IC}=$ intervalo de confianza, $\mathrm{IAM}=$ infarto agudo del miocardio, $\mathrm{FA}=$ fibrilación auricular, $\mathrm{POP}=$ postoperatorio, $\mathrm{IVU}=$ infección de vías urinarias

El promedio de estancia UCI fue 6.1 días con un rango de 1-65 días. El promedio de estancia hospitalaria fue 12.7 días con un rango de 3-87 días.

\section{Valoración de calidad de vida y funcionalidad}

A la pregunta que es equivalente a la autopercepción en salud: ¿Se siente usted mejor, igual o peor que antes de la cirugía de corazón abierto? El 79.4\% consideraban que se sentían mejor. El promedio del componente de salud física fue inferior al de la población de referencia (43.7 vs 49.6), y los factores de riesgo relacionados con menor calidad de salud física fueron sexo femenino ( 58.1 vs $35.6 \mathrm{p}<0.0001)$ y mayor edad, el comportamiento con relación a la edad fue el siguiente: 60-64 años (50.7), 65-69 (48.7), 70-75 (34.5) y $\operatorname{los} \geq 80$ años (31.5) con una $\mathrm{p}<0.03$ (Figura 5).

De todas las comorbilidades la única que tuvo tendencia a menor puntaje en el componente de salud física fue la diabetes mellitus tipo $2(\mathrm{p}=0.07)$.

Con respecto al componente de salud mental el comportamiento del promedio estuvo por encima del promedio de la población de referencia (53.2 vs 49.3) (Figura 5); y no se encontró ninguna variable que se relacionara con menor calidad del componente de salud mental.

En la valoración de la funcionalidad $94.1 \%$ de los pacientes evaluados eran independientes para su $\mathrm{ABC}$ físico

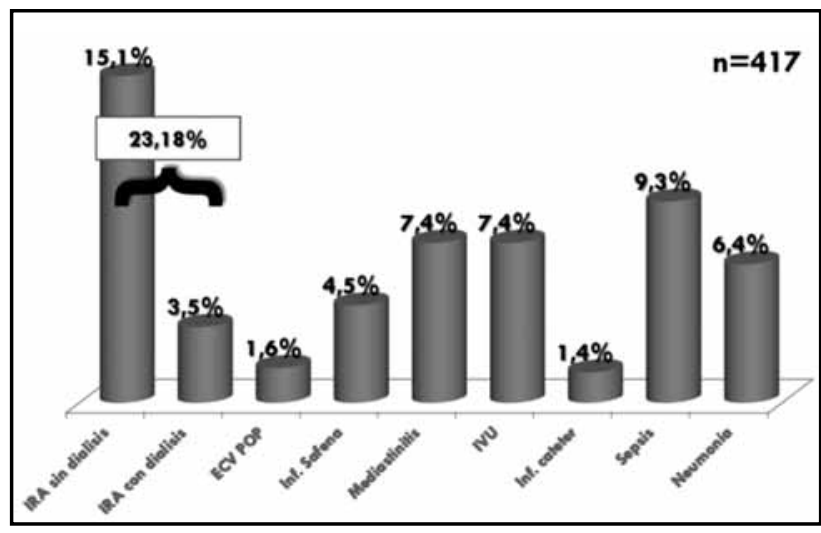

Figura 2 Complicaciones postoperatorias; IRA=insuficiencia renal aguda, ECV=evento cerebrovascular, $I V U=$ infección de vías urinarias.

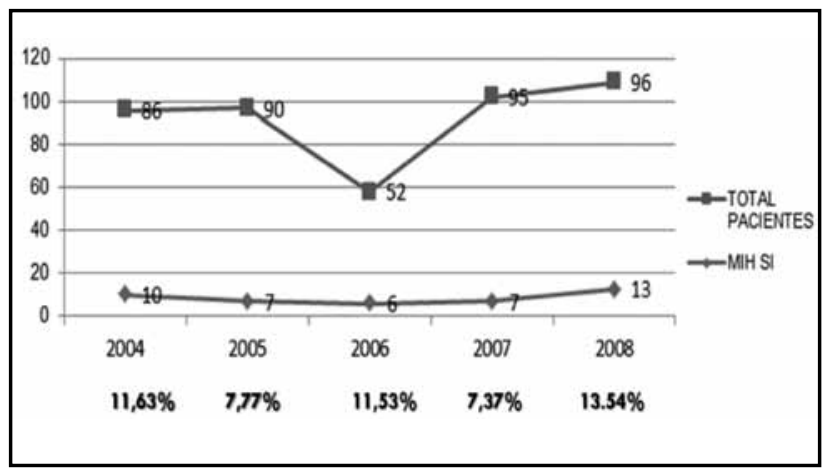

Figura 3. Mortalidad por años de cirugía. MIH: mortalidad intrahospitalaria.

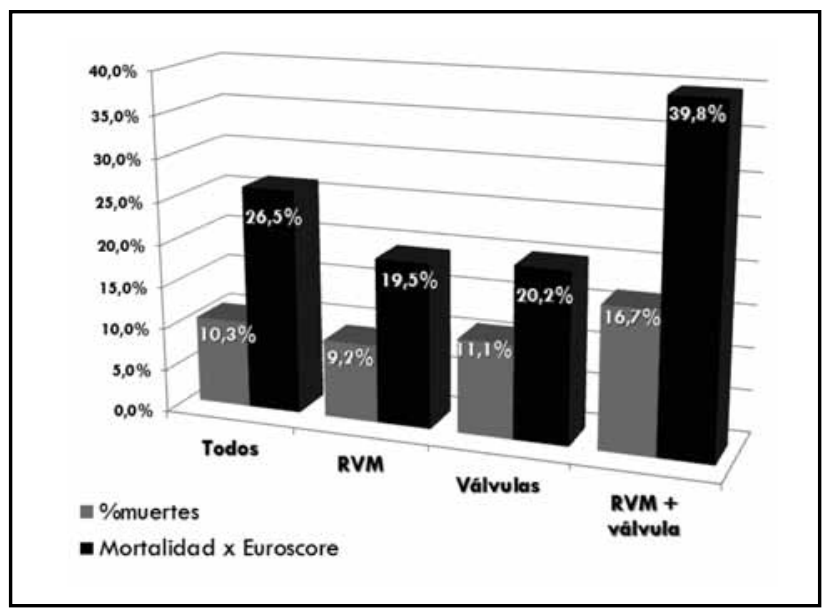

Figura 4. Mortalidad real vs mortalidad esperada por EUROSCORE. RVM= revascula rización miocárdica.

medido por escala de Barthel (independencia $\geq 80$ puntos). Mientras que en $A B C$ instrumental medido por LAWTON modificado versión 8-30,85.3\% consideraban que requerían algún tipo de ayuda; las variables ir de compras y salir de casa mostraron menor funcionalidad en el ABC instrumental en sexo femenino, actividades que requieren mejor desplazamiento y desempeño social (Figura 6). 


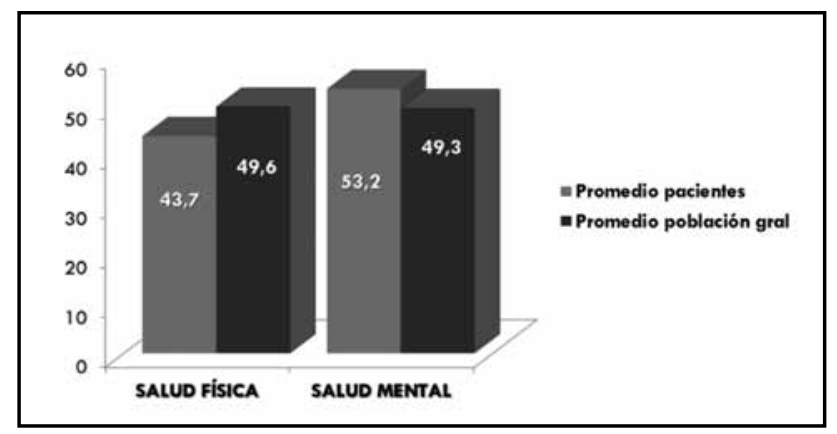

Figura 5. Calidad de vida medida Short Form 12 HealthSurveyversion 2 (SF-12v2).

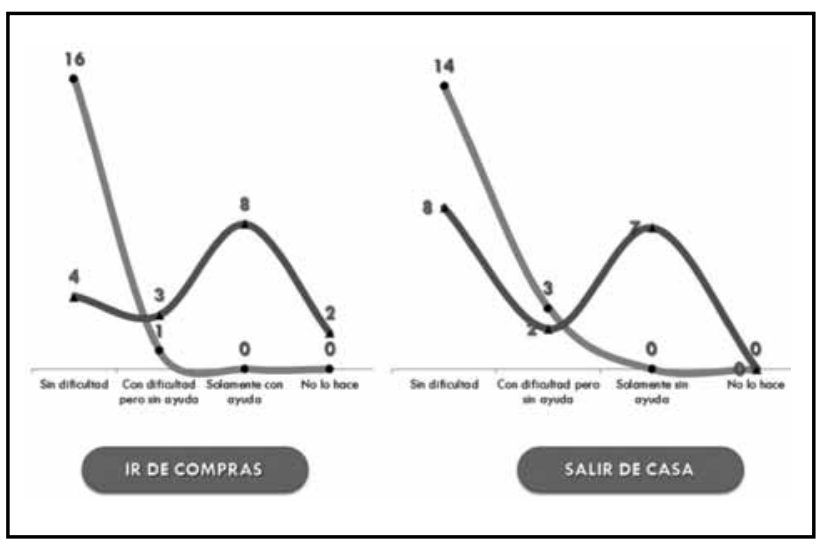

Figura 6. Características del Lawton que más se afectaron en la valoración del ABC instrumental posterior a cirugía cardiaca.

El bajo porcentaje de diligenciamiento de las variables socio-demográficas no permitió un análisis al respecto.

\section{Discusión}

Se describen las características de una cohorte de pacientes ancianos sometidos a cirugía cardiovascular, donde se encontró un mayor número de hombres sometidos a cirugía de corazón abierto, con una relación de 1.6:1 y un promedio de edad de 68.6 años que es comparativamente menor al de la mayoría de estudios referenciados (6, 12-16, 23). Con un alto porcentaje de pacientes con factores de riesgo cardiovascular que se correlaciona con lo descrito en otros estudios, incluido un estudio anterior de esta misma institución donde se describe la epidemiología del infarto agudo de miocardio (17).

La mortalidad global es comparativamente similar a la de otras publicaciones las cuales muestran rangos entre $4.1 \%$ y $10.7 \%(6,14-16,18-20,24)$ siendo factores influyentes los previamente conocidos como son el mayor compromiso de la función ventricular, la peor clase funcional, la carga de la comorbilidad y el número alto de cirugías realizadas con criterios de urgencia en una institución que fue el único centro de referencia del Eje Cafetero durante el periodo de tiempo evaluado. Al igual que en otros grupos de cirugía cardiaca los promedios descritos de la mortalidad pronosticada por EUROSCORE logístico son mayores que los obtenidos (6,
$15,18,25)$. Es de esperarse que la presentación de complicaciones sean fuertes predictores de mortalidad de los que resaltamos: la falla renal que requirió soporte dialítico $\mathrm{OR}=$ 11.9 (IC 95\%: 4.1-35), el bajo gasto postoperatorio $\mathrm{OR}=5.3$ (IC 95\%: 2.7-10.3), el infarto perioperatorio $\mathrm{OR}=5.0$ (IC 95\%: 2.2-11.1), la sepsis OR=4.9 (IC 95\%:2.2-10.7), el sangrado que requirió reintervención $\mathrm{OR}=4.1$ (IC 95\%:1.98.8) y la falla respiratoria $\mathrm{OR}=3.4$ (IC 95\%:1.6-7.2) con unos amplios intervalos de confianza que reflejan la colinearidad de éstos, así como la compleja interacción de los múltiples factores por tener en cuenta.

Es importante destacar la mejoría en la autopercepción en salud donde $79.4 \%$ referían sentirse mejor que antes del procedimiento quirúrgico, lo que justifica que la cirugía cardiaca cuando los síntomas deterioran la calidad de vida.

El comportamiento de la calidad de vida es similar al encontrado en otros estudios, donde existe un menor puntaje del componente de salud física $(6,20-22,26)$, sólo un estudio encontró que los promedios posterior a la cirugía cardiaca se encontraban superiores a los de la población de referencia (22); el sexo femenino y la edad se comportaron como factores de riesgo para peor calidad de vida postoperatoria aclarando que los altos puntajes del componente de salud mental es una constante en todos los estudios ya referenciados $(6,20-22,26)$.

Los pocos estudios que evalúan funcionalidad hace difícil la comparación con otros grupos, lo que sí llama la atención es el nivel de independencia que se mantiene para las actividades básicas cotidianas físicas (Barthel >80 puntos), en comparación con las actividades instrumentales en las cuales tenemos que resaltar las dos actividades más comprometidas del Lawton en el grupo de las mujeres (salir de casa y hacer compras), con lo cual podríamos plantear como hipótesis un mayor compromiso cardiopulmonar como limitante de la movilidad que en ultimas es un componente fundamental para desarrollarlas, eso sí, sin dejar de lado la no valoración dentro del estudio de otros factores relacionados como son el componente osteomuscular que bien podría haber influenciado en este resultado.

Dentro de las debilidades del estudio encontramos: su característica retrospectiva y descriptiva, ser un estudio unicéntrico, la falta de diligenciamiento en las fuentes de datos de las variables epidemiológicas y el hecho de no poder contactar todos los pacientes vivos para valorar la funcionalidad y calidad de vida.

\section{Conclusiones}

Este es el primer estudio que describe la epidemiología de un grupo de pacientes ancianos llevados a cirugía cardiaca; esto es importante para poder dar a conocer si se están cumpliendo los objetivos y para mejorar día a día en la atención. Las estadísticas antes mencionadas son comparables con las de otros estudios a nivel mundial.

Importante destacar la mejoría de la autopercepción en salud posterior al procedimiento, donde la gran mayoría de 
pacientes evaluados se sentían mejor que antes de la cirugía. Se evidencia una significativa preservación del ABC físico evaluado por Barthel con una puntuación muy cercana a la población de referencia en el promedio del componente, de salud física medida por SF-12v2, lo que se correlaciona con los hallazgos del Lawton al valorar funcionalidad.

Todo lo anterior implica que en el manejo postoperatorio la rehabilitación no sólo física sino mental es muy importante para lograr los objetivos trazados desde el punto de vista de la funcionalidad y calidad de vida; por esto la propuesta de una evaluación integral geriátrica y multidimensional aplicada a estos pacientes puede mejor los resultados. Faltarían estudios prospectivos y de seguimiento que evalúen las variables aquí estudiadas, principalmente la calidad de vida y la funcionalidad posterior a la cirugía de corazón abierto en grandes cohortes de octogenarios que en el momento son el grupo de interés a nivel internacional.

\section{Referencias}

1. DANE . Proyecciones departamentales y nacionales de población. Departamento Administrativo Nacional de Estadísticas DANE disponible en www. dane.gov.co

2. Majeed F, Kelem MD. Acute Coronary Syndromes in the Elderly, Clin Geriatr Med 2007; (23): 425-440.

3. Yazdanyar A, Newman AB. The Burden of Cardiovascular Disease in the Elderly: Morbidity, Mortality, and Costs. Clin Geriatr Med 2009; (25): 563-577.

4. Ribera Casado José M. Cirugía cardiaca y edad avanzada, Servicio de Geriatría Hospital Clínico San Carlos. Universidad Complutense. Madrid. España. Rev Esp Cardiol 2008; 61(6): 564-6.

5. Conti V, Lick SD. Cardiac Surgery: Indications and Management Options to Optimize Ouctcomes. Clin Geriatr Med 2006; (22): 559-574.

6. Ghanta RK, Sheka PS, McGurk S. Long-Term Survival and Quality of Life Justify Cardiac Surgery in the Very Elderly Patient. Ann Thorac Surg 2011; (92): 851-7.

7. Gandek B, Ware JE, Aaronson NK, Apolone G, Bjorner JB, Brazier JE et al. Cross-Validation of item Selection and scoring for the SF-12 Health Survey in Nine Contries: Results from the IQOLA Project. J Clin Epidemiol 1998; (Vol 51): 1171-1178

8. Ramirez R, Agredo RA, Jerez AM, Chapal L. Calidad de Vida y Condiciones de Salud en Adultos Mayores no Institucionalizados en Cali, Colombia. Rev Salud Publica 2008; 10 (4): 529; 536.

9. SF-36.org. The SF-12 survey: An even Shorter Health Survey. Disponible en http://www.sf-36.org/tools/sf12. shtml\#version2.

10. Lawton MP, Brody EM. Assessment of older people: self maintaining and instrumental activities of daily living. Gerontologist 1969; (9): 179-86.
11. Kane RA, Kane RL. 1993. Evaluación de las necesidades en los ancianos. Barcelona: Fundación Caja Madrid, SG Editores: 36-67.

12. Wilson MF, Baig MK, and Ashraf H. Quality of Life in Octogenarians After Coronary Artery Bypass Grafting. Am J Cardiol 2005; (95): 761-764.

13. Permanyer C, Miralda, Brotons C, Ribera A, Caballero JA, Cascant P et al. Resultados después de cirugía coronaria: determinantes de calidad de vida relacionada con la salud postoperatoria; RevEspCardiol 2001; (54): 607-616.

14. Speziale G, Nasso G, Barattoni MC et al. Operative and Middle-Term Results of Cardiac Surgery in Nonagenarians. Circulation 2010; (121): 208-213.

15. Zingone B, Gatti G, Rauber E, Tiziani P, Dreas L, Pappalardo A, Benussi B, Spina A. Early and Late Outcomes of Cardiac Surgery in Octogenarians. Ann Thorac Surg 2009; (87): 71-8.

16. Alexander KP, Anstrom KJ, Muhlbaier LH, Grosswald RD, Smith PK, Jones RH et al. Outcomes of Cardiac Surgery in Patients Age $\geq 80$ Years: Results from the National Cardiovascular Network. Journal of the American College of Cardiology; 35 (3): 731-738.

17. Cano N. Epidemiologia del infarto agudo del miocardio en el Hospital Santa Sofía de Manizales. Estudio descriptivo (junio de 2000 a junio de 2003). Rev Col Cardiol 2004; (11)3: 157-163.

18. López-Rodríguez FJ, González-Santos JM, Dalmau MJ. Cirugía cardiaca en el anciano: comparación de resultados a medio plazo entre octogenarios y ancianos de 75 a 79 años. Rev Esp Cardiol 2008; (61): 579-88.

19. Huber CH, Goeber V, Berdat P, Carrel T, Eckstein F. Benefits of cardiac surgery in octogenarians - a postoperative quality of life assessment. Eur J Cardiothor Surg 2007; (31): 1099-105.

20. Fruitman DS, MacDougal CE, Ross DB. Cardiac Surgery in Octogenarians: Can Elderly Patients Benefit? Quality of Life After Cardiac Surgery. Ann Thorac Surg 1999; (68): $2129-35$

21. Miralda CP, Cuixart CB, Ribera A, Caballero JA, Castello PC, Moral I. Resultados después de cirugía coronaria: determinantes de calidad de vida relacionada con la salud postoperatoria. Rev Esp Cardiol 2001; (54): 607-616.

22. Kurlansky PA, Williams DB, Traad EA, Carrillo RG, Schor JS, Zucker M et al. Arterial Grafting results in reduced Mortality and Enhanced Long-Term Quality of Life in Octogenarians. Ann Thorac Surg 2003; (76): 418-427.

23. Rosborough D. Cardiac Surgery in elderly patients. Critical Care Nurse 2006 (26): 24-32

24. Ravi KG, Srem SS, Siobhan McG, Rosborough D, and Sary FA. Nonelec tive cardiac surgery in the elderly: Is it justified?.J Thorac Cardiovasc Surg 2010; (140): 103-9.

25. Mohamed Y, Thomas R, Norman J. Perioperative determinants of morbidity and mortality in elderly patients undergoing cardiac surgery. Crit Care Med 2001; 29(9).

26. Lafuente S, Antoni T, Laia Br, González Ra, Bertrán MJ. Validación de modelo probabilístico EuroSCORE en pacientes intervenidos de injerto coronario. Rev Esp Cardiol 2008; 61(6): 589-94.

27. Fruitman DS, MacDougall CE, Ross, DB. Cardiac Surgery in Octogenarians: Can Elderly Patients Benefit? Quality of Life After Cardiac Surgery. Ann Thorac Surg 1999; (68): $2129-35$. 